\title{
Database Design on Teaching Management System Based on SQL Server
}

\author{
Zhe Li $i^{1, a}$, Houhua Shen ${ }^{2, b}$ \\ ${ }^{1}$ College of International Exchange, Bohai University, Jinzhou, 121013, China \\ ${ }^{2}$ College of Engineering, Bohai University, Jinzhou, 121013, China \\ a503412508@qq.com, bshenhouhuashh@126.com
}

\begin{abstract}
Keywords: SQL Server; teaching management system; database design; conceptual structure design; logical structure design; index design

Abstract. The network teaching system in higher education is playing an increasingly important role in modernization construction. Through the network teaching, creating a digital learning environment, to promote the reform of the education concept, teaching content and method, improve the teaching quality of education and improve students' ability of survival and development in the information society. The research content of this article to provide technical support for the teaching management system development. Based on the SQL Server database management system structure, conceptual structure design, logic structure design and the index design. In this paper, design of the database with reasonable structure, storage efficiency, as well as good independence, for the teaching management system development provides a feasible solution.
\end{abstract}

\section{Introduction}

Network education in the higher education modernization is playing an increasingly important role. Through the network teaching, creating a digital learning environment, to promote the reform of the education concept, teaching content and method, to improve the teaching quality and efficiency of education, to cultivate learners have good social culture and values, to improve learners' learning ability and the ability of survival and development in the information society. In the implementation of the network teaching, support teaching activity teaching platform integrates all kinds of network teaching application subsystem and tools, the technical basis of network teaching. Although both at home and abroad has developed many network teaching platform software, but from the perspective of the function and application effect of the software is still in the exploratory stage.

In the face of scientific and technological progress and the rapid development of social economy, our country's higher education from the aspects such as management system, teaching mode, curriculum reconstruction are facing serious challenges, there are many don't adapt. From the school's point of view, in order to make students master more knowledge, should offer more courses for the students. But as the knowledge explosion, colleges and universities is generally restricted by limited study time with students total teaching hours. To this end, many schools to carry out network teaching, hoping to use the network teaching system to speed up the knowledge transfer. At present the application of network teaching system, the general is the teaching information network, realize the teaching process automation, but under the traditional teaching mode and management system, the role of network teaching system has not fully realized. Specific performance in school between repeat construction, course many problems, such as lack of reconstruction and integration.

Basic work is the database construction of teaching management system development, only the construction of the reasonable and efficient database system, to develop a perfect information management system. Database is basic work of the construction of the database design, database design is for a given application environment, constructing the optimal database model, establish a database and application systems, to store data effectively and meet the application requirements of various users. Database design is to establish a database and its application technology in the system, is the core technology in information system development and Suggestions. This article is based on 
SQL Server database management system for database design, to provide technical support for the teaching management system development.

\section{Database Management System Structure of SQL Server}

SQL Server is Microsoft's relational database management system. SQL Server is a comprehensive database platform, using the integrated business intelligence tools provides enterprise data management. SQL Server database engine for relational data and structured data provides a more secure and reliable storage capabilities, to build and manage for the business of high availability and high performance data application. SQL Server database management system structure is shown in Fig. 1.

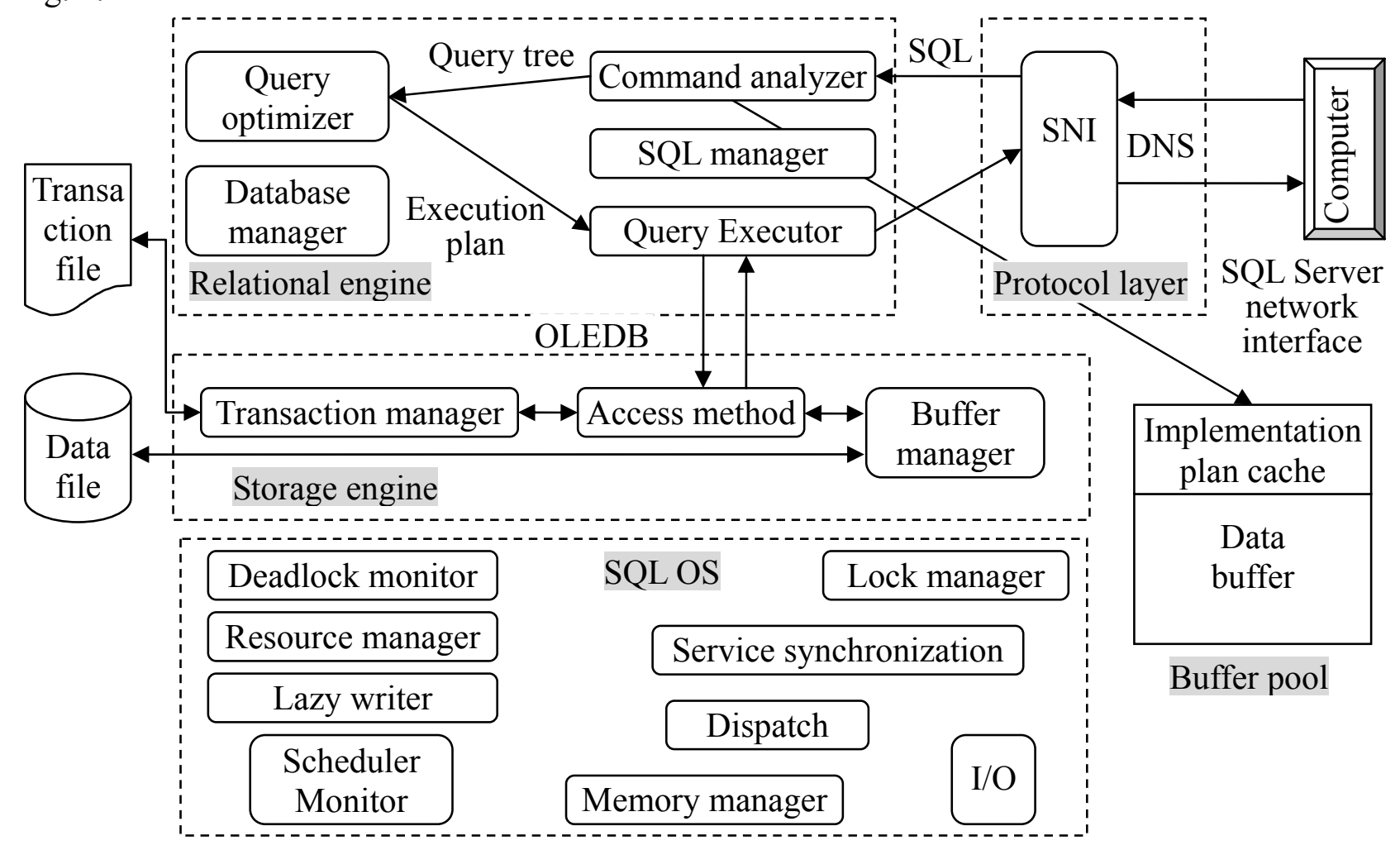

Fig. 1. Database management system structure of SQL Server

Data engine is the core of data management solutions [1]. The database engine is used for storage, processing, and protect the data of core services. Using the database engine can control access and quickly handle affairs, so as to satisfy the business enterprise inside very demanding and requires application need to process large amounts of data. Using the database engine created for online transaction processing and on-line analytical processing data of relational database. Including the creation of tables used to store data and used to view, manage and protect the data security of database objects. Can use the SQL Server Management Studio Management database objects, using SQL Server Profiler capture Server events.

SQL Server has the following advantages: easy to use, suitable for the scalability of distributed organization, for the data warehouse decision support functions, and is closely related to many other Server software integration, the good performance-to-price ratio, etc. Bring the flexibility for data management and analysis, allows the unit to calmly respond to rapidly changing environment, so as to gain a competitive advantage. From the perspective of the data management and analysis of business intelligence and make full use of original data can be converted to Web brings the opportunity is very important. As a complete package database and data analysis, the SQL Server for the rapid development of a new generation of enterprise core business applications, for enterprises to win competition advantage to open the door to victory. As an important benchmark for scalability and speed record holder, SQL Server is a completely Web support database products, 
provides the support for the core of the extensible markup language (XML), and on the Internet and the ability to query outside the firewall.

\section{Conceptual Structure Design on Database}

Conceptual structure design is the most commonly used tool E-R Diagram (Entity Relationship Diagram), E-R Diagram provides the said entities, attributes, and contact method, is used to describe the conceptual model of the real world[2]. Constitute the basic elements of E-R diagram are entities, attributes, and contact, the entity with rectangular, said the box to fill in real name; Attributes expressed with rounded shape, form the box to fill in the property name, and undirected edges with the corresponding physical connection; Contact with diamond, diamond box to fill in contact name, with no physical connection to the relevant side respectively with, at the same time in undirected edge near the contact type. Combining with the results of other researchers [3, 4], the concept of system database structure design results are shown in Fig. 2.

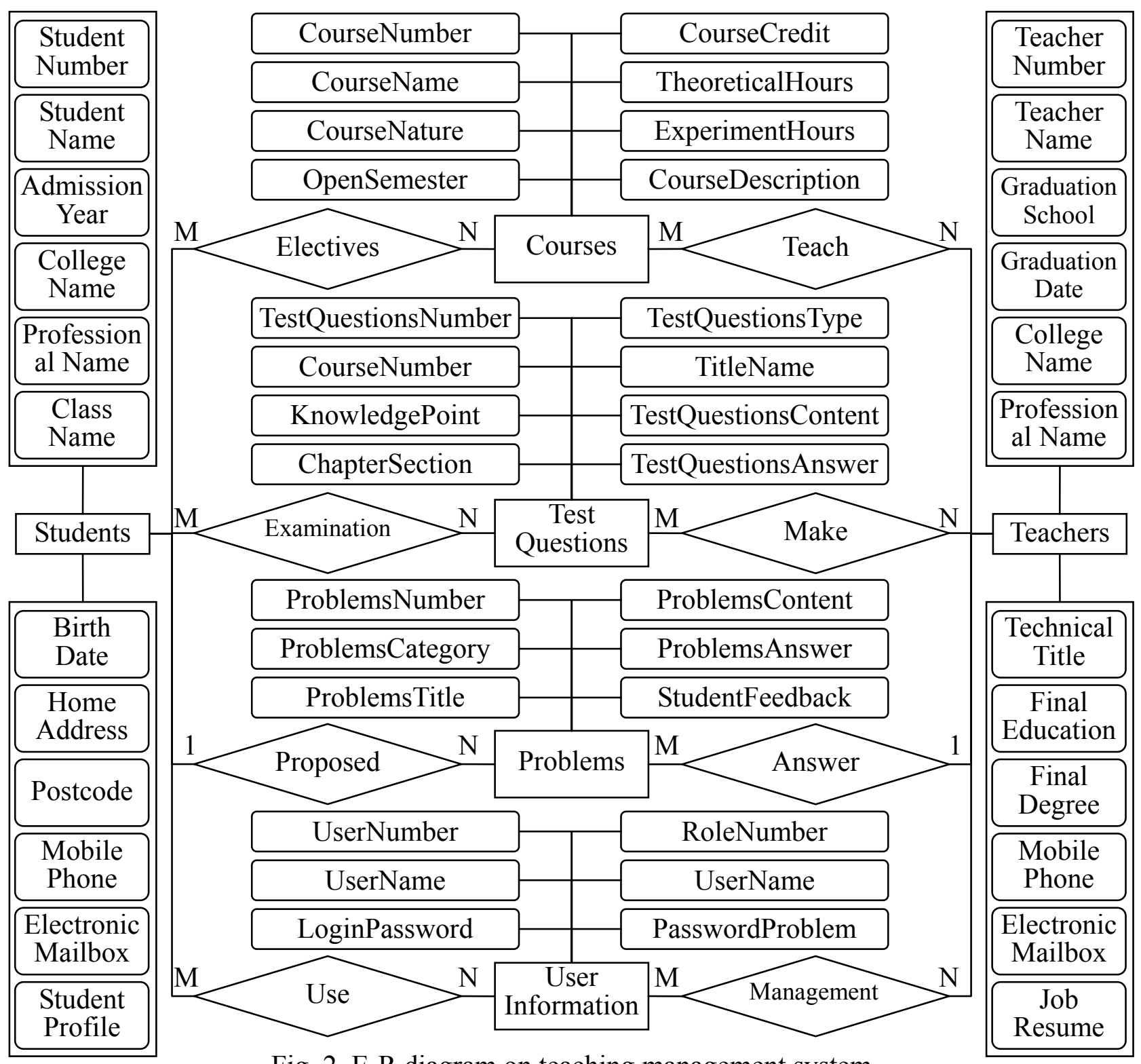

Fig. 2. E-R diagram on teaching management system

As can be seen from the figure 2, there are six entity system, respectively is "Students, Teachers, Courses, Test Questions, Problems, User Information"; Six found two contact with a one-to-many relationship between entities and six many-to-many connection, two one-to-many links were "Proposed, Answer", 2 one-to-many link are "Electives, Teach, Examination, Make, Use, Management". 


\section{Logical Structure Design on Database}

In order to establish a database of the user's requirements, the need to convert the conceptual model to a particular DBMS support data model. Its task is to convert the conceptual model to particular DBMS support data model, the process of the need to consider the characteristics of the specific data model and the performance of DBMS [5]. Teaching management system database structure design results are shown in Table 1.

Table 1. Logical Structure Design on teaching management system

\begin{tabular}{|c|c|c|c|c|c|c|c|}
\hline \multicolumn{4}{|c|}{ "Students" Table } & \multicolumn{4}{|c|}{ "Teachers" Table } \\
\hline 1 & StudentNumber & char & 10 & 51 & TeacherNumber & char & 10 \\
\hline 2 & StudentName & varchar & 20 & 52 & TeacherName & varchar & 20 \\
\hline 3 & AdmissionYear & char & 4 & 53 & GraduationSchool & varchar & 30 \\
\hline 4 & CollegeName & varchar & 50 & 54 & GraduationDate & datetime & 4 \\
\hline 5 & ProfessionalName & varchar & 30 & 55 & CollegeName & varchar & 50 \\
\hline 6 & ClassName & varchar & 10 & 56 & ProfessionalName & varchar & 30 \\
\hline 7 & BirthDate & datetime & 4 & 57 & TechnicalTitle & varchar & 20 \\
\hline 8 & HomeAddress & varchar & 100 & 58 & FinalDegree & varchar & 20 \\
\hline 9 & Postcode & char & 6 & 59 & FinalEducation & varchar & 20 \\
\hline 10 & MobilePhone & varchar & 20 & 60 & MobilePhone & varchar & 20 \\
\hline 11 & ElectronicMailbox & varchar & 50 & 61 & ElectronicMailbox & varchar & 50 \\
\hline 12 & StudentProfile & text & 8 & 62 & JobResume & text & 8 \\
\hline \multicolumn{4}{|c|}{ "Courses" Table } & \multicolumn{4}{|c|}{ "TestQuestions" Table } \\
\hline 21 & CourseNumber & char & 10 & 71 & TestQuestionsNumber & char & 10 \\
\hline 22 & CourseName & varchar & 40 & 72 & CourseNumber & char & 10 \\
\hline 23 & CourseNature & varchar & 40 & 73 & KnowledgePoint & varchar & 50 \\
\hline 24 & OpenSemester & char & 1 & 74 & ChapterSection & varchar & 20 \\
\hline 25 & CourseCredit & decimal & 4,1 & 75 & TestQuestionsType & varchar & 20 \\
\hline 26 & TheoreticalHours & smallint & 2 & 76 & TitleName & varchar & 100 \\
\hline 27 & ExperimentHours & smallint & 2 & 77 & TestQuestionsContent & text & 8 \\
\hline 28 & CourseDescription & text & 8 & 78 & TestQuestionsAnswer & text & 8 \\
\hline \multicolumn{4}{|c|}{ "Problems" Table } & \multicolumn{4}{|c|}{ "UserInformation" Table } \\
\hline 31 & ProblemsNumber & char & 10 & 81 & UserNumber & char & 10 \\
\hline 32 & ProblemsCategory & varchar & 20 & 82 & UserName & varchar & 40 \\
\hline 33 & ProblemsTitle & varchar & 100 & 83 & LoginPassword & varchar & 20 \\
\hline 34 & ProblemsContent & text & 8 & 84 & RoleNumber & char & 10 \\
\hline 35 & ProblemsAnswer & text & 8 & 85 & RoleName & varchar & 40 \\
\hline 36 & StudentFeedback & text & 8 & 86 & PasswordProblem & varchar & 100 \\
\hline \multicolumn{4}{|c|}{ "Electives" Table } & \multicolumn{4}{|c|}{ "Teach" Table } \\
\hline 41 & StudentNumber & char & 10 & 91 & CourseNumber & char & 10 \\
\hline 42 & CourseNumber & char & 10 & 92 & TeacherNumber & char & 10 \\
\hline \multicolumn{4}{|c|}{ "Proposed" Table } & \multicolumn{4}{|c|}{ "Answer" Table } \\
\hline 56 & StudentNumber & char & 10 & 96 & TeacherNumber & char & 10 \\
\hline 57 & ProblemsNumber & char & 10 & 97 & ProblemsNumber & char & 10 \\
\hline 58 & ProposedTime & datetime & 4 & 98 & AnswerTime & datetime & 4 \\
\hline
\end{tabular}




\section{Index Design on Database}

When no index, accessed columns from the table to get specific criteria will conduct a full table scan line data operation, the server process will all the data in the table of loaded into memory, then the line by line matching, then the SQL execution time is mainly consumed on the table data loading and data matching, need to consume large amounts of I/o and CPU costs. Index is one or more of a database table column value sort structure, create indexes can improve system performance. By creating a unique index, ensure the uniqueness of each row of data in a database; Accelerate the speed of data retrieval, this also is to create an index of the main reason; To accelerate the connection between the table and table, especially in the implementation is particularly meaningful reference data completeness; In the use of grouping and sorting clause for data retrieval, also significantly reduce the query time of grouping and sorting; By using the index in the process of query optimization of hidden, improve system performance $[6,7]$.

In this system, each table to create a clustering index, and according to the query need to design more than clustering index. "Students" table, for example, according to the "StudentNumber" field to create the cluster index; Press "StudentName", "AdmissionYear", "CollegeName", "ProfessionalName", "ClassName" five fields such as creating a clustering index.

\section{Conclusion}

Compared with traditional teaching, network teaching system has an irreplaceable advantage, improve the network teaching system can directly replace the traditional teaching more all the process of teaching in a network. Advantages in the following aspects: learning form is novel, loved by students. The diversity of learning, meet the curiosity of students; Diversification of learning content, meet the students desire for knowledge; The randomness, learning problems can be solved in a timely manner; Learning convenience, students choose the most effective way to learn; Fairness into the network learning system to study, you can enjoy equal opportunity to learn; Teacher force strong, guarantee the authority of learning content; Parents and teachers is a good helper to help teachers, parents, students to solve their own problems. In this paper, based on SQL Server database design, for the teaching management system development provides a feasible solution to strengthen the teaching management in colleges and universities, improve the level of education informatization is of great significance.

\section{References}

[1]Baidu Encyclopedia, "Microsoft Server," http://baike.baidu.com/link?url=qOXbUKKUkb6vFEsDxOISiDT77YOF29Ta, 2015-11-25.

[2] Z. Y. Duan, "Database Design Methodology," Journal of Nanchang College of Education, vol. 20, no. 4, pp. 85-86, 2005.

[3] H. Liu, "Database design and application of teaching management system in Higher Vocational Colleges," Chinese \& Foreign Entrepreneurs, vol. 32, no. 8, pp. 161-162, 2015.

[4] Y. Zhou, "Design and implementation of teaching management system based on ASP.NET," Office Informatization, vol. 11, no. 6, pp. 58-60, 2015.

[5] Baidu Encyclopedia, "Logical Structure Design on Database," http://baike.baidu.com/link?url=UE8qIaMokFIGIDM-p6YJr-BCm76oXuYPxp5fbzCO6pPG2S BSIYE1u8C9veROPjm7qbLyzppWilC8cyiKuZvc6_, 2015-11-25.

[6] X. Chen, "The establishment and analysis of index in Server SQL," Scientific and Technological Information, vol. 29, no. 36, pp. 288-289, 2012.

[7] Z. S. Yang, C. Zhang, "The impact of the index on the execution cost of SQL," Information Communication, vol. 27, no. 1, pp. 136-137, 2013. 